\title{
Solutions for PDEs with constant coefficients and derivability of functions ranged in commutative algebras
}

\section{Anatoliy Pogorui ${ }^{\mathrm{a}}$, Ramón M. Rodríguez-Dagnino ${ }^{\mathrm{b} *}$ and Michael Shapiro}

\author{
Communicated by W. Sprößig
}

Given a PDE with real or complex partial derivatives and with constant coefficients, we propose a method of assigning to it a set of algebra-valued functions in such a manner that the components of the latter are solutions of the PDE. Copyright (c) 2013 John Wiley \& Sons, Ltd.

Keywords: monogenic functions; partial differential equations; commutative algebra

\section{Introduction}

It is well known that the real and imaginary parts of any holomorphic function are harmonic functions of two variables. In this paper, we extend this idea to finite-dimensional commutative algebras; that is, we prove that if some basis of a subspace of a commutative algebra satisfies a polynomial equation, then the components of a monogenic function on the subspace are solutions of the respective partial differential equation (PDE). We illustrate these concepts with a few examples.

An algebra of analytical functions of complex variables is associated with the two-dimensional Laplace equation; that is, the real and imaginary components of an analytic function are solutions of the two-dimensional Laplace equation. The algebra of functions $\vec{f}: \mathbb{R}^{3} \rightarrow \mathbb{R}^{\infty}$, which is associated with the three-dimensional Laplace equation, was introduced by Ketchum [1,2]. The so-called biharmonic bases in commutative algebras and monogenic functions on these algebras associated with the biharmonic equation are studied in $[3,4]$. By using a similar technique, we study the biwave equation in Section 4.3. An interesting solution of the threedimensional Laplace equation has been elaborated in [5] by defining a related commutative and associative algebra over the field of complex numbers, and other related developments can be consulted in [6-8]. In this work, we generalize these ideas.

In the paper [9], we study a one-dimensional random motion having a general $m$-Erlang distribution for the sojourn times. Let $f(t, x)$ be the probability density function (PDF) of a particle position at time $t$, provided that it exists. We obtained the following higher order hyperbolic equations for $f(t, x)$ :

$$
\left(\frac{\partial}{\partial t}-v \frac{\partial}{\partial x}+\lambda\right)^{m}\left(\frac{\partial}{\partial t}-v \frac{\partial}{\partial x}+\lambda\right)^{m} f(t, x)-\lambda^{2 m} f(t, x)=0
$$

where $v>0$ is the velocity of the particle and $\lambda$ is the parameter of the $m$-Erlang distribution. It is assumed that a particle started at $x=0$ and, hence, $f(0, x)=\delta(x)$. For $m=1$, this equation is the well-known telegraph equation, and its solution has been obtained by using Riemann's method. For $m=2$, we have the so-called bi-telegraph equation, and up to our best knowledge, its solution is unknown. By using our method, we obtain the solution up to the initial conditions. We also describe the algorithm for solving of PDEs with non-constant coefficients.

\section{Differentiability on finite-dimensional commutative algebras}

Let $\mathbf{A}$ be a finite-dimensional commutative unitary algebra over $K=\mathbb{R}$ (or $\mathbb{C}$ ); assume that the set of vectors $\vec{e}_{0}, \vec{e}_{1}, \ldots, \vec{e}_{n}$ is a basis of $\mathbf{A}$ and that $\vec{e}_{0}$ is the unit of this algebra. Suppose that $\mathbf{B}$ is an $(m+1)$-dimensional subspace of $\mathbf{A}$ with the basis $\vec{e}_{0}, \vec{e}_{1}, \ldots, \vec{e}_{m}, m \leq n$. Any function $\vec{f}: \mathbf{B} \rightarrow \mathbf{A}$ is of the form

\footnotetext{
${ }^{a}$ Department of Applied Mathematics, Zhytomyr State University, Zhytomyr, Ukraine

${ }^{b}$ DIEC, Campus Monterrey, Tecnológico de Monterrey (ITESM), 64849 Monterrey, Nuevo Leon, Mexico

'Escuela Superior de Física y Matemáticas, Instituto Politécnico Nacional, Mexico D. F. 07738, Mexico

*Correspondence to: Ramón M. Rodríguez-Dagnino, DIEC, Campus Monterrey, ITESM, 64849 Monterrey, Nuevo Leon, Mexico.

${ }^{\dagger}$ E-mail:rmrodrig@itesm.mx
} 


$$
\vec{f}(\vec{x})=\sum_{k=0}^{n} \vec{e}_{k} u_{k}(\vec{x}),
$$

where $u_{k}(\vec{x})=u_{k}\left(x_{0}, x_{1}, \ldots, x_{m}\right)$ are $\mathbb{R}$-valued (or $\mathbb{C}$-valued) functions of $m+1$ variables $x_{i} \in K$.

Definition 1. $\vec{f}(\vec{x})$ is called differentiable at a point $\vec{x}_{0} \in \mathbf{B}$ if there exists a unique element $\vec{f}^{\prime}\left(\vec{x}_{0}\right) \in \mathbf{A}$ such that for any $\vec{h} \in \mathbf{B}$,

$$
\vec{h} \vec{f}^{\prime}\left(\vec{x}_{0}\right)=\lim _{\varepsilon \rightarrow 0} \frac{\vec{f}\left(\vec{x}_{0}+\varepsilon \vec{h}\right)-\vec{f}\left(\vec{x}_{0}\right)}{\varepsilon}
$$

It should be kept in mind that $\varepsilon \in K$ in accordance with the algebra $\mathbf{A}$.

The existence of the limit at the right-hand side is equivalent to the condition that the map $f: \mathbf{B} \longrightarrow \mathbf{A}$ is Gateaux-differentiable along any direction $\vec{h}$. In addition, this definition includes another quite strong condition: the Gateaux derivative along $\vec{h}$ is proportional to $\vec{h}$. In the case when the algebra $\mathbf{A}$ is over $\mathbb{C}$, Definition 1 implies Gateaux differentiability along complex lines.

Furthermore, the proportionality coefficient is the same for any direction $\vec{h}$. It is denoted by $\vec{f}^{\prime}\left(\vec{x}_{0}\right)$ and called as the derivative of $\vec{f}$ at the point $\vec{x}_{0}$. For $\mathbf{A}=\mathbf{B}=\mathbb{C}$, this definition is also equivalent to the (complex) differentiability of the complex function $\vec{f}$, and $\vec{f}^{\prime}\left(\vec{x}_{0}\right)$ becomes the usual complex derivative.

Thus, one can expect that the introduced functions inherit some important properties of holomorphic functions.

We say that $\vec{f}: \mathbf{B} \longrightarrow \mathbf{A}$ is differentiable (in $\mathbf{B}$ ) or monogenic if it is differentiable at any point of $\mathbf{B}$.

Definition 1 includes the existence of all $(m+1)$ partial derivatives. Indeed, it is enough to take $h=e_{k}, k \in\{0,1,2, \ldots, m\}$. Moreover, it implies

$$
\frac{\partial \vec{f}}{\partial x_{k}}\left(\vec{x}_{0}\right)=e_{k} \vec{f}^{\prime}\left(\vec{x}_{0}\right)
$$

for all $k$ but with the same coefficient $\vec{f}^{\prime}\left(\vec{x}_{0}\right)$.

It turns out that Equation (1) is equivalent to the definition of differentiability.

Theorem 1

A function $\vec{f}(\vec{x})=\sum_{k=0}^{n} \vec{e}_{k} u_{k}(\vec{x})$ is differentiable at $\vec{x}_{0}$ if and only if it has all partial derivatives $\frac{\partial \vec{f}}{\partial x_{k}}\left(\vec{x}_{0}\right)$ proportional, with the same proportionality coefficient, to the respective basis vectors $\vec{e}_{k}$ :

$$
\frac{\partial \vec{f}}{\partial x_{k}}\left(\vec{x}_{0}\right)=\vec{e}_{k} \alpha\left(\vec{x}_{0}\right)
$$

whenever it holds

$$
\alpha\left(\vec{x}_{0}\right)=\vec{f}^{\prime}\left(\vec{x}_{0}\right)
$$

Proof

The necessary part is given already. The sufficient part is much the same as in [10], and we give it briefly for completeness. It follows from Equation (3) that

$$
\begin{gathered}
\alpha\left(\vec{x}_{0}\right)=\frac{\partial \vec{f}}{\partial x_{0}}=\sum_{k=0}^{n} \vec{e}_{k} \frac{\partial u_{k}}{\partial x_{0}}, \\
\vec{e}_{1} \alpha\left(\vec{x}_{0}\right)=\frac{\partial \vec{f}}{\partial x_{1}}=\sum_{k=0}^{n} \vec{e}_{k} \frac{\partial u_{k}}{\partial x_{1}}=\vec{e}_{1} \sum_{k=0}^{n} \vec{e}_{k} \frac{\partial u_{k}}{\partial x_{0}}, \\
\vdots \\
\vec{e}_{n} \alpha\left(\vec{x}_{0}\right)=\frac{\partial \vec{f}}{\partial x_{n}}=\sum_{k=0}^{n} \vec{e}_{k} \frac{\partial u_{k}}{\partial x_{n}}=\vec{e}_{n} \sum_{k=0}^{n} \vec{e}_{k} \frac{\partial u_{k}}{\partial x_{0}} .
\end{gathered}
$$

Let $\vec{h}=\sum_{k=0}^{n} h_{k} \vec{e}_{k}$, then it follows from Equation (5) 


$$
\begin{aligned}
\vec{h} \alpha\left(\vec{x}_{0}\right) & =\sum_{k=0}^{n} h_{k} \vec{e}_{k} \alpha\left(\vec{x}_{0}\right) \\
& =h_{0} \sum_{k=0}^{n} \vec{e}_{k} \frac{\partial u_{k}}{\partial x_{0}}+h_{1} \vec{e}_{1} \sum_{k=0}^{n} \vec{e}_{k} \frac{\partial u_{k}}{\partial x_{0}}+\cdots+h_{n} \vec{e}_{n} \sum_{k=0}^{n} \vec{e}_{k} \frac{\partial u_{k}}{\partial x_{0}} \\
& =h_{0} \sum_{k=0}^{n} h_{k} \vec{e}_{k} \frac{\partial u_{k}}{\partial x_{0}}+h_{1} \sum_{k=0}^{n} h_{k} \vec{e}_{k} \frac{\partial u_{k}}{\partial x_{1}}+\cdots+h_{n} \sum_{k=0}^{n} h_{k} \vec{e}_{k} \frac{\partial u_{k}}{\partial x_{n}} \\
& =\lim _{\varepsilon \rightarrow 0} \frac{\vec{f}\left(\vec{x}_{0}+\varepsilon \vec{h}\right)-\vec{f}\left(\vec{x}_{0}\right)}{\varepsilon} .
\end{aligned}
$$

Thus, we conclude that $\vec{f}$ is differentiable and $\alpha\left(\vec{x}_{0}\right)=\vec{f}^{\prime}\left(\vec{x}_{0}\right)$.

Equation (5) implies the following Cauchy-Riemann type of conditions for a differentiable function $\vec{f}$ :

$$
\begin{gathered}
\sum_{k=0}^{n} \vec{e}_{k} \frac{\partial u_{k}}{\partial x_{1}}=\vec{e}_{1} \sum_{k=0}^{n} \vec{e}_{k} \frac{\partial u_{k}}{\partial x_{0}}, \\
\sum_{k=0}^{n} \vec{e}_{k} \frac{\partial u_{k}}{\partial x_{2}}=\vec{e}_{2} \sum_{k=0}^{n} \vec{e}_{k} \frac{\partial u_{k}}{\partial x_{0}}, \\
\vdots \\
\sum_{k=0}^{n} \vec{e}_{k} \frac{\partial u_{k}}{\partial x_{m}}=\vec{e}_{m} \sum_{k=0}^{n} \vec{e}_{k} \frac{\partial u_{k}}{\partial x_{0}},
\end{gathered}
$$

or in the vector form

$$
\frac{\partial \vec{f}}{\partial x_{k}}=\vec{e}_{k} \frac{\partial \vec{f}}{\partial x_{0}}, \quad k=0,1, \ldots, m
$$

Remark 1. It is easily verified that Theorem 1 remains true even for $\mathbf{B}$ with basis $\vec{e}^{\prime}{ }_{1}, \vec{e}^{\prime}{ }_{2}, \ldots, \vec{e}^{\prime}{ }_{m+1}$ that does not contain the unit $\vec{e}_{0}$ but having an invertible element among its basis vectors. In this case, the Cauchy-Riemann type of conditions can be stated as follows:

$$
\frac{\partial \vec{f}}{\partial x_{k}}=\vec{e}_{k}^{\prime} \vec{e}_{l}^{\prime}{ }_{l}^{-1} \frac{\partial \vec{f}}{\partial x_{l}}, \quad k, I=0,1, \ldots, m \text {. }
$$

\section{Differentiable functions providing solutions to PDEs}

Consider the two-dimensional Laplace equation

$$
\Delta_{2} u=\frac{\partial^{2} u}{\partial x_{0}^{2}}+\frac{\partial^{2} u}{\partial x_{1}^{2}}=0
$$

where $u$ is an $\mathbb{R}$-valued function of the two real variables $x_{0}$ and $x_{1}$. Of course, Equation (8) makes sense even for functions $u$ taking values in a normed space, but we should remark that Equation (8) has a 'real nature' in a completely understandable meaning. At the same time, it is trivial to say that solutions of Equation (8) can be provided by purely complex tools; for example, let $f(z)=u_{0}(z)+i u_{1}(z)$ be a holomorphic function of the variable $z=x_{0}+i x_{1}$, then the functions $u_{0}$ and $u_{1}$ are solutions of Equation (8). That is, the real PDE (8) has a commutative algebra on the complex numbers $\mathbb{C}$ such that complex holomorphic functions provide real solutions to it.

We will extend this idea to much more general situations. For given integers $m, r \geq 1$, let

$$
P\left(\xi_{0}, \xi_{1}, \ldots, \xi_{m}\right):=\sum_{i_{0}+i_{1}+\cdots+i_{m}=r} C_{i_{0}, i_{1}, \ldots, i_{m}} \xi_{0}^{i_{0}} \xi_{1}^{i_{1}} \ldots \xi_{m}^{i_{m}}
$$

where $C_{i_{0}, i_{1}, \ldots, i_{m}}$ are constant coefficients. Consider the following PDE

$$
P\left(\partial_{0}, \partial_{1}, \ldots, \partial_{m}\right)\left[u\left(x_{0}, x_{1}, \ldots, x_{m}\right)\right]=0 .
$$

where $\partial_{k}:=\frac{\partial^{k}}{\partial x^{k}}$. 
Theorem 2

Let $P$ be a polynomial as in Equation (10). Let a function $\vec{f}: \mathbf{B} \longrightarrow \mathbf{A}$ and its derivatives $\vec{f}^{\prime}, \vec{f}^{\prime \prime}, \ldots, \overrightarrow{f^{r}}$ be differentiable, $\vec{f}(\vec{x})=\sum_{k=0}^{n} \overrightarrow{e_{k}} u_{k}(\vec{x})$. Assume that the basis $\vec{e}_{0}, \vec{e}_{1}, \ldots, \vec{e}_{m}$ of the subspace $\mathbf{B}$ of the algebra $\mathbf{A}$ is such that

$$
P\left(\vec{e}_{0}, \vec{e}_{1}, \ldots, \vec{e}_{m}\right)=0
$$

then the functions $u_{k}(\vec{x}), k=0,1, \ldots, n$ are solutions of Equation (10)

Proof

It follows from the Cauchy-Riemann type condition (7) that

$$
\frac{\partial^{i_{n}} \vec{f}}{\partial x_{k}^{i_{n}}}=\left(\vec{e}_{k}\right)^{i_{n}} \frac{\partial \vec{f}}{\partial x_{0}}, \quad k=0,1, \ldots, m
$$

This implies, for $i_{0}+i_{1}+\ldots+i_{m} \leq r$, that

$$
\frac{\partial^{i_{0}+i_{1}+\ldots+i_{m} \vec{f}}}{\partial x_{0}^{i_{0}} \partial x_{1}^{i_{1}} \ldots \partial x_{m}^{i_{m}}}=\left(\vec{e}_{0}\right)^{i_{0}}\left(\vec{e}_{1}\right)^{i_{1}} \ldots\left(\vec{e}_{m}\right)^{i_{m}} \frac{\partial^{i_{0}+i_{1}+\ldots+i_{m}} \vec{f}}{\partial x_{0}^{i_{0}+i_{1}+\ldots+i_{m}}} .
$$

Therefore, we obtain

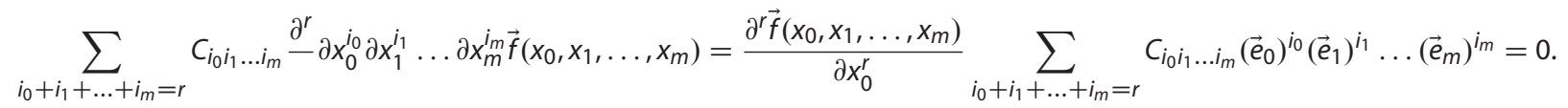

Hence, it follows that every component $u_{k}(\vec{x}), k=0,1, \ldots, n$, of the function $\vec{f}$ is a solution of Equation (10).

Remark 2. Similarly to Theorem 1, Theorem 2 remains true even if $\mathbf{B}$ does not contain the unit $\vec{e}_{0}$, but among its basis vectors, there is an invertible element.

\section{Examples}

\subsection{The Laplace equation}

Given a polynomial $P$ as in Equation (10), Theorem 2 suggests to look for non-real solutions of Equation (11). For instance, for the two-dimensional Laplace equation, Equation (8), one has that $P\left(\xi_{0}, \xi_{1}\right)=\xi_{0}^{2}+\xi_{1}^{2}$; hence, Equation (11) becomes

$$
e_{0}^{2}+e_{1}^{2}=0
$$

that is, $1+e_{1}^{2}=0$, and we arrive predictably at the field $\mathbb{C}$ of complex numbers. It is easy to see that, given a function $f=u+i v: \mathbb{C} \longrightarrow \mathbb{C}$, it is differentiable in a sense of Definition 1 if it is holomorphic. And, of course, its components $u, v$ provide solutions to Equation (8).

\subsection{The wave equation}

It is given by

$$
\left(\frac{\partial^{2}}{\partial x^{2}}-\frac{\partial^{2}}{\partial y^{2}}\right) u(x, y)=0
$$

Thus, we have $P\left(\xi_{0}, \xi_{1}\right)=\xi_{0}^{2}-\xi_{1}^{2}$. Hence, Equation (11) becomes

$$
1^{2}-e^{2}=0
$$

because we are interested in non-real solutions, then we arrive to the algebra of hyperbolic numbers $\mathbf{D}=\left\{a_{0}+a_{1} e \mid a_{0}, a_{1} \in \mathbb{R}\right\}$, where $e^{2}=1, e \neq \pm 1$; see [11].

A function $f(x, y)=u(x, y)+e v(x, y)$, where $u, v: \mathbb{R}^{2} \rightarrow \mathbb{R}$, is differentiable if the following Cauchy-Riemann conditions are fulfilled:

$$
\begin{gathered}
\frac{\partial u}{\partial x}(x, y)=\frac{\partial v}{\partial y}(x, y) \\
\frac{\partial}{\partial y} u(x, y)=\frac{\partial}{\partial x} v(x, y) .
\end{gathered}
$$

Therefore, the functions $u, v$ are solutions to the wave equation. 
For hyperbolic functions $f=u+e v$, an analogue of the complex holomorphic function theory is known, see [11], where the derivative of $f$ is introduced in the usual way; that is, if $z=x+e y, h=h_{1}+e h_{2}$, then

$$
f^{\prime}\left(z_{0}\right)=\lim _{h \rightarrow 0} \frac{f\left(z_{0}+h\right)-f(z)}{h}
$$

without intersecting zero divisors of $\mathbb{D}$. The function $f$ is hyperbolic holomorphic (that is, it has a derivative everywhere) if and only if

$$
\frac{\partial f}{\partial \bar{z}}:=\left(\frac{\partial f}{\partial x}+e \frac{\partial f}{\partial y}\right)=0
$$

which is equivalent to Equation (15). Thus, the differentiability in hyperbolic number's case turns out to be equivalent to a more classic approach.

Remark 3. Using hyperbolic numbers, the wave operator in Equation (14) admits a factorization

$$
\frac{\partial^{2}}{\partial x^{2}}-\frac{\partial^{2}}{\partial y^{2}}=\left(\frac{\partial}{\partial x}+e \frac{\partial}{\partial y}\right)\left(\frac{\partial}{\partial x}-e \frac{\partial}{\partial y}\right),
$$

in a similar manner as the Laplace operator in complex case.

\subsection{The biwave equation}

The biwave equation is given by

$$
\left(\frac{\partial^{2}}{\partial x^{2}}-\frac{\partial^{2}}{\partial y^{2}}\right)^{2} u(x, y)=0
$$

and it has been obtained in [9] in the context of random evolutions and studied in [12]. In this case, we have $P\left(\xi_{0}, \xi_{1}\right)=\left(\xi_{0}^{2}-\xi_{1}^{2}\right)^{2}$.

It is easy to see that for this case, we can consider the algebra of dual numbers [13], $\mathbf{P}=\left\{a_{0}+a_{1} \epsilon \mid a_{0}, a_{1} \in \mathbb{R}\right\}$, where $\epsilon^{2}=0$.

According to Theorem 2, we have to find a basis $\vec{e}_{1}, \vec{e}_{2}$ such that $\left(\vec{e}_{1}^{2}-\vec{e}_{2}^{2}\right)^{2}=\overrightarrow{0}$. For this purpose, the case $\vec{e}_{1}^{2}-\vec{e}_{2}^{2} \neq \overrightarrow{0}$ results interesting.

Let us define $\vec{e}_{1}=\alpha_{1}+\alpha_{2} \epsilon$ and $\vec{e}_{2}=\beta_{1}+\beta_{2} \epsilon$, then we obtain

$$
\vec{e}_{1}^{2}=\alpha_{1}^{2}+2 \alpha_{1} \alpha_{2} \epsilon, \quad \vec{e}_{2}^{2}=\beta_{1}^{2}+2 \beta_{1} \beta_{2} \epsilon .
$$

Now, suppose that $\vec{e}_{1}^{2}-\vec{e}_{2}^{2}=\epsilon$. Then, it follows from Equation (18) that

$$
\begin{aligned}
\alpha_{1} & = \pm \beta_{1} \\
\alpha_{1}\left(\beta_{2} \pm \alpha_{2}\right) & =\frac{1}{2},
\end{aligned}
$$

or

$$
\begin{aligned}
& \vec{e}_{1}=\alpha_{1}+\alpha_{2} \epsilon \\
& \vec{e}_{2}= \pm\left[\alpha_{1}+\left(\alpha_{2}-\frac{1}{2 \alpha_{1}}\right)\right] .
\end{aligned}
$$

Assume a function $\vec{f}: \mathbf{P} \rightarrow \mathbf{P}$, such that

$$
\vec{f}(x, y)=u_{1}(x, y) \vec{e}_{1}+u_{2}(x, y) \vec{e}_{2}, \quad x, y \in \mathbb{R} .
$$

In accordance to Remark 1, the function $\vec{f}(x, y)$ is monogenic if

$$
\vec{e}_{1} \frac{\partial}{\partial y} u_{1}(x, y)+\vec{e}_{2} \frac{\partial}{\partial y} u_{2}(x, y)=\vec{e}_{2} \vec{e}_{1}^{-1}\left(\vec{e}_{1} \frac{\partial}{\partial x} u_{1}(x, y)+\vec{e}_{2} \frac{\partial}{\partial x} u_{1}(x, y)\right) .
$$

For the particular case $\alpha_{1}=\alpha_{2}=1$, we have $\vec{e}_{1}=1+\epsilon, \vec{e}_{2}=1+\epsilon / 2$, and consequently

$$
\begin{aligned}
\frac{\partial}{\partial y} u_{1}(x, y)+\frac{\partial}{\partial y} u_{2}(x, y) & =\frac{\partial}{\partial x} u_{1}(x, y)+\frac{\partial}{\partial x} u_{2}(x, y) ; \\
\frac{\partial}{\partial y} u_{1}(x, y)+\frac{1}{2} \frac{\partial}{\partial y} u_{2}(x, y) & =\frac{1}{2} \frac{\partial}{\partial x} u_{1}(x, y) .
\end{aligned}
$$

If we consider distinct real numbers $\alpha_{1} \neq 0$ and $\alpha_{2}$, then we can obtain different systems of first-order linear PDEs whose solutions are solutions of the biwave equation (17). 
4.4. The three-dimensional Laplace equation

The three-dimensional Laplace equation is given by

$$
\left(\frac{\partial^{2}}{\partial x^{2}}+\frac{\partial^{2}}{\partial y^{2}}+\frac{\partial^{2}}{\partial z^{2}}\right) u(x, y, z)=0
$$

This equation implies the polynomial $P\left(\xi_{0}, \xi_{1}, \xi_{2}\right)=\xi_{0}^{2}+\xi_{1}^{2}+\xi_{2}^{2}$, and Equation (11) has the following view $1+e_{1}^{2}+e_{2}^{2}=0$. In this case, we can use the bicomplex algebra $\mathbf{B}=\left\{a_{0}+a_{1} i+a_{2} j+a_{3} e \mid a_{i} \in \mathbb{R}\right\}$.

It is easy to see that $1+\frac{1}{2} i^{2}+\frac{1}{2} j^{2}=0$. So, we should consider $\mathbf{B}$ with the basis $\vec{e}_{0}=1, \vec{e}_{1}=\frac{1}{\sqrt{2}} i, \vec{e}_{2}=\frac{1}{\sqrt{2}} j$, and $\vec{e}_{3}=\frac{1}{\sqrt{2}} e$.

Now, suppose $\mathbf{B}_{0}=\left\{a_{0} \vec{e}_{0}+a_{1} \vec{e}_{1}+a_{2} \vec{e}_{2} \mid a_{k} \in \mathbb{R}\right\}$ is a subspace of $\mathbf{B}$.

Consider a function $\vec{f}: \mathbf{B}_{o} \rightarrow \mathbf{B}$, namely,

$$
\vec{f}(x, y, z)=u_{0}(x, y, z)+u_{1}(x, y, z) \frac{1}{\sqrt{2}} i+u_{2}(x, y, z) \frac{1}{\sqrt{2}} j+u_{3}(x, y, z) \frac{1}{\sqrt{2}} e
$$

where $x, y, z \in \mathbb{R}$ and $u_{k}: \mathbb{R}^{3} \rightarrow \mathbb{R}, k=0,1,2,3$.

According to Theorem 2, if the function $\vec{f}$ is monogenic, then $u_{k}(x, y, z)$ are solutions of Equation (19).

So, let us write the Cauchy-Riemann type of conditions for $\vec{f}$ :

$$
\begin{aligned}
\frac{\partial}{\partial y} u_{0}(x, y, z)+ & \frac{1}{\sqrt{2}} i \frac{\partial}{\partial y} u_{1}(x, y, z)+\frac{1}{\sqrt{2}} j \frac{\partial}{\partial y} u_{2}(x, y, z)+\frac{1}{\sqrt{2}} e \frac{\partial}{\partial y} u_{3}(x, y, z) \\
& =\frac{1}{\sqrt{2}} i \frac{\partial}{\partial x} u_{0}(x, y, z)-\frac{1}{2} \frac{\partial}{\partial x} u_{1}(x, y, z)+\frac{1}{2} e \frac{\partial}{\partial x} u_{2}(x, y, z)-\frac{1}{2} j \frac{\partial}{\partial x} u_{3}(x, y, z) \\
\frac{\partial}{\partial z} u_{0}(x, y, z)+ & \frac{1}{\sqrt{2}} i \frac{\partial}{\partial z} u_{1}(x, y, z)+\frac{1}{\sqrt{2}} j \frac{\partial}{\partial z} u_{2}(x, y, z)+\frac{1}{\sqrt{2}} e \frac{\partial}{\partial z} u_{3}(x, y, z) \\
& =\frac{1}{\sqrt{2}} j \frac{\partial}{\partial x} u_{0}(x, y, z)+\frac{1}{\sqrt{2}} e \frac{\partial}{\partial x} u_{1}(x, y, z)-\frac{\partial}{\partial x} u_{2}(x, y, z)-\frac{1}{\sqrt{2}} i \frac{\partial}{\partial x} u_{3}(x, y, z)
\end{aligned}
$$

Therefore, we have four pairs of equations:

1. $\frac{\partial}{\partial y} u_{0}(x, y, z)=-\frac{1}{2} \frac{\partial}{\partial x} u_{1}(x, y, z), \quad \frac{\partial}{\partial y} u_{1}(x, y, z)=\frac{\partial}{\partial x} u_{0}(x, y, z)$;

2. $\frac{\partial}{\partial y} u_{2}(x, y, z)=-\frac{1}{\sqrt{2}} \frac{\partial}{\partial x} u_{3}(x, y, z), \quad \frac{\partial}{\partial y} u_{3}(x, y, z)=\frac{1}{\sqrt{2}} \frac{\partial}{\partial x} u_{2}(x, y, z)$;

3. $\frac{\partial}{\partial z} u_{0}(x, y, z)=-\frac{\partial}{\partial x} u_{2}(x, y, z), \quad \frac{\partial}{\partial z} u_{2}(x, y, z)=\frac{\partial}{\partial x} u_{0}(x, y, z)$;

4. $\frac{\partial}{\partial z} u_{1}(x, y, z)=-\frac{\partial}{\partial x} u_{3}(x, y, z), \quad \frac{\partial}{\partial z} u_{3}(x, y, z)=\frac{\partial}{\partial x} u_{1}(x, y, z)$.

It is interesting to observe that cases (3) and (4) are Cauchy-Riemann conditions with respect to the corresponding variables. For instance, case (3) is a Cauchy-Riemann condition for $u_{0}(x, y, z)$ and $u_{1}(x, y, z)$ with respect to the variables $x$ and $z$. Hence, we obtain the two-dimensional Laplace equations

$$
\begin{aligned}
& \frac{\partial^{2}}{\partial x^{2}} u_{0}(x, y, z)+\frac{\partial^{2}}{\partial z^{2}} u_{0}(x, y, z)=0 \\
& \frac{\partial^{2}}{\partial x^{2}} u_{2}(x, y, z)+\frac{\partial^{2}}{\partial z^{2}} u_{2}(x, y, z)=0 .
\end{aligned}
$$

Furthermore, it follows from case (1) that

$$
\begin{aligned}
& \frac{\partial^{2}}{\partial x^{2}} u_{0}(x, y, z)+2 \frac{\partial^{2}}{\partial y^{2}} u_{0}(x, y, z)=0 \\
& 2 \frac{\partial^{2}}{\partial x^{2}} u_{1}(x, y, z)+\frac{\partial^{2}}{\partial y^{2}} u_{1}(x, y, z)=0 .
\end{aligned}
$$

In addition, from case (2), we have

$$
\begin{aligned}
& \frac{\partial^{2}}{\partial x^{2}} u_{2}(x, y, z)+2 \frac{\partial^{2}}{\partial y^{2}} u_{2}(x, y, z)=0 \\
& \frac{\partial^{2}}{\partial x^{2}} u_{3}(x, y, z)+2 \frac{\partial^{2}}{\partial y^{2}} u_{3}(x, y, z)=0 .
\end{aligned}
$$


Much in the same way, we can study the four-dimensional Laplace equation.

Now, let us consider the example of a complex differential equation.

4.4.1. The two-dimensional complex wave equation. The two-dimensional complex wave equation is given by

$$
\left(\frac{\partial^{2}}{\partial z_{1}^{2}}-\frac{\partial^{2}}{\partial z_{2}^{2}}\right) u\left(z_{1}, z_{2}\right)=0
$$

where $z_{1}, z_{2}$ are complex. Thus, we have $P\left(\xi_{0}, \xi_{1}\right)=\xi_{0}^{2}-\xi_{1}^{2}$, and Equation (11) has the following view $1-e_{1}^{2}=0$. For this case, we can use the bicomplex algebra as a two-dimensional commutative algebra over $\mathbb{C}$ of the following presentation $\{a+e b\}$, where $a, b \in \mathbb{C}$, $e^{2}=1$, ie $=e i$. By stating that $e_{1}=e$, we obtain a function $f\left(z_{1}, z_{2}\right)=u\left(z_{1}, z_{2}\right)+e v\left(z_{1}, z_{2}\right)$, where $u, v: \mathbb{C}^{2} \rightarrow \mathbb{C}$, is monogenic if the following Cauchy-Riemann conditions are fulfilled:

$$
\begin{aligned}
\frac{\partial}{\partial z_{1}} u\left(z_{1}, z_{2}\right) & =\frac{\partial}{\partial z_{1}} v\left(z_{1}, z_{2}\right) \\
\frac{\partial}{\partial z_{2}} u\left(z_{1}, z_{2}\right) & =\frac{\partial}{\partial z_{1}} v\left(z_{1}, z_{2}\right) .
\end{aligned}
$$

We should recall that these are complex derivatives.

\section{Infinite-dimensional case}

Let us consider the case when $\mathbf{A}$ is an infinite-dimensional commutative unital algebra over $K$, with the basis $e_{k}, k=0,1,2 \ldots$, and $\mathbf{B}$ is an $(m+1)$-dimensional subspace of $\mathbf{A}$ (similarly to the finite-dimensional case).

Now, consider a function $f: \mathbf{B} \rightarrow \mathbf{A}$ of the following form

$$
f(\vec{x})=\sum_{k=0}^{\infty} u_{k}(\vec{x}) e_{k}
$$

where $u_{k}(\vec{x})=u_{k}\left(x_{0}, x_{1}, \ldots, x_{m}\right)$ are real or complex functions.

Similarly to Theorem 1 , it can be proved that the function $f$ is monogenic if and only if

$$
\frac{\partial f}{\partial x_{k}}=e_{k} \frac{\partial f}{\partial x_{0}}, \quad k=0,1,2, \ldots
$$

\subsection{Example: Fourier algebra}

Suppose that $\mathbf{A}$ is the $\mathbb{R}$-algebra of Fourier series with the basis $e_{k}=\cos k t, k=0,1,2, \ldots$, and $\mathbf{B}$ is the following subspace $\mathbf{B}=\left\{\sum_{k=0}^{m} x_{k} e_{k}, x_{k} \in \mathbb{R}\right\}$. It is easily verified that $e_{k} e_{m}=\frac{1}{2}\left(e_{k-m}+e_{k+m}\right), k \geq m$.

The Cauchy-Riemann type conditions for this case follows from Equation (21)

$$
\frac{\partial u_{0}}{\partial x_{1}}=\frac{1}{2} \frac{\partial u_{1}}{\partial x_{0}}, \quad \frac{\partial u_{1}}{\partial x_{1}}=\frac{\partial u_{0}}{\partial x_{0}}+\frac{1}{2} \frac{\partial u_{2}}{\partial x_{0}},
$$

and

$$
\frac{\partial u_{k}}{\partial x_{1}}=\frac{1}{2}\left(\frac{\partial u_{k-1}}{\partial x_{0}}+\frac{\partial u_{k+1}}{\partial x_{0}}\right), \quad k=2,3, \ldots ;
$$

similarly,

$$
\begin{gathered}
\frac{\partial u_{0}}{\partial x_{2}}=\frac{1}{2} \frac{\partial u_{2}}{\partial x_{0}}, \quad \frac{\partial u_{1}}{\partial x_{2}}=\frac{1}{2}\left(\frac{\partial u_{1}}{\partial x_{0}}+\frac{\partial u_{3}}{\partial x_{0}}\right) \\
\frac{\partial u_{2}}{\partial x_{2}}=\frac{\partial u_{0}}{\partial x_{0}}+\frac{1}{2} \frac{\partial u_{4}}{\partial x_{0}}, \quad \text { and } \quad \frac{\partial u_{k}}{\partial x_{2}}=\frac{\partial u_{k-2}}{\partial x_{0}}+\frac{1}{2} \frac{\partial u_{k+2}}{\partial x_{0}}, \quad k=2,3, \ldots ;
\end{gathered}
$$

and so on.

Now, let us consider the case when $m=1$, hence $\mathbf{B}=\{x+y \cos t, x, y \in \mathbb{R}\}$. Then,

$$
f(w)=f(x+y \cos t)=u_{0}(x, y)+\sum_{k=1}^{\infty} u_{k}(x, y) \cos k t .
$$


Therefore,

$$
u_{k}(x, y)=\frac{2}{\pi} \int_{0}^{\pi} f(x+y \cos t) \cos k t d t
$$

and the Cauchy-Riemann conditions can be written as

$$
\frac{\partial u_{0}}{\partial y}=\frac{1}{2} \frac{\partial u_{1}}{\partial x}, \quad \frac{\partial u_{1}}{\partial y}=\frac{\partial u_{0}}{\partial x}+\frac{1}{2} \frac{\partial u_{2}}{\partial x}, \quad \frac{\partial u_{k}}{\partial y}=\frac{1}{2}\left(\frac{\partial u_{k-1}}{\partial x}+\frac{\partial u_{k+1}}{\partial x}\right), k=2,3, \ldots
$$

In order to find the solutions for $u_{0}$ and $u_{1}$, let us define

$$
p(x, y):=u_{0}(x, y) \quad \text { and } \quad q(x, y):=\frac{y u_{1}(x, y)}{2} .
$$

Hence, we have

$$
y \frac{\partial p(x, y)}{\partial y}=\frac{\partial q(x, y)}{\partial x}
$$

Next, let us show that

$$
\frac{\partial u_{2}}{\partial x}=\frac{\partial u_{1}}{\partial y}-\frac{u_{1}}{y} .
$$

Indeed, taking into account Equation (21), we have to show that

$$
\frac{2}{\pi} \int_{0}^{\pi} f^{\prime}(w) \cos 2 t d t-\frac{2}{\pi} \int_{0}^{\pi} f^{\prime}(w) \cos ^{2} t d t+\frac{2}{y \pi} \int_{0}^{\pi} f(w) \cos t d t=0 .
$$

Integrating by parts, we have

$$
\frac{2}{y \pi} \int_{0}^{\pi} f(w) \cos t d t=\frac{2}{\pi} \int_{0}^{\pi} f^{\prime}(w) \sin ^{2} t d t
$$

that proves Equation (23).

Substituting Equation (24) in the second equation of the Cauchy-Riemann conditions, we have

$$
\frac{\partial u_{1}}{\partial y}-2 \frac{\partial u_{0}}{\partial x}+\frac{u_{1}}{y}=0
$$

Whence

$$
\frac{\partial}{\partial y}\left(\frac{2}{y} q(x, y)\right)-2 \frac{\partial p(x, y)}{\partial x}+\frac{2}{y^{2}} q(x, y)=0
$$

or

$$
y \frac{\partial p(x, y)}{\partial x}=\frac{\partial q(x, y)}{\partial y}
$$

It follows from Equations (24) and (25) that

$$
y\left(\frac{\partial^{2} p(x, y)}{\partial x^{2}}-\frac{\partial^{2} p(x, y)}{\partial y^{2}}\right)-\frac{\partial p(x, y)}{\partial y}=0
$$

and

$$
y\left(\frac{\partial^{2} q(x, y)}{\partial x^{2}}-\frac{\partial^{2} q(x, y)}{\partial y^{2}}\right)+\frac{\partial q(x, y)}{\partial y}=0 .
$$

Thus, by defining $f(w)=e^{w}=e^{x+y \cos t}$, we obtain the following solution for Equation (26)

$$
p(x, y)=e^{x} \int_{0}^{\pi} e^{y \cos t} d t
$$

and

$$
q(x, y)=e^{x} \int_{0}^{\pi} e^{y \cos t} \cos t d t
$$

for Equation (27). 


\section{The bi-telegraph equation}

Consider the following equation

$$
\left(\frac{\partial^{2}}{\partial t^{2}}-\frac{\partial^{2}}{\partial y^{2}}\right)^{2} g_{c}(t, y)-\lambda^{4} g_{c}(t, y)=0
$$

This equation is called the bi-telegraph equation, and it was obtained in [9] after finding the distribution of the position of a particle that moves on the line driven by a point process with two-Erlang sojourn times.

By applying the transformation $f(t, x)=e^{\lambda t} g(t, x)$ and changing the variable $y=\frac{x}{v}$, we reduce Equation (28) to

$$
\left(\frac{\partial^{2}}{\partial t^{2}}-\frac{\partial^{2}}{\partial y^{2}}\right)^{2} \mathbf{f}(t, y, z)-\frac{\partial^{4}}{\partial z^{4}} \mathbf{f}(t, y, z)=0 .
$$

We will seek for solutions of Equation (29) by using the theory of differentiable functions on commutative algebras.

Let $\mathbf{A}_{0}$ be a four-dimensional commutative algebra over $\mathbb{R}$, assume that the set $\left\{e_{0}, e_{1}, e_{2}, e_{3}\right\}$ is a basis of $\mathbf{A}_{0}$ with the Cayley table,

$$
e_{i} e_{j}=e_{i \oplus j}
$$

where $i \oplus j=i+j(\bmod 4)$.

The algebra $\mathbf{A}_{0}$ has the following matrix representation:

$$
e_{k} \rightarrow P_{k}=P_{1}^{k}
$$

where the basis $e_{I}=\mathbf{e}^{\prime}, I=0,1,2,3$, and $\mathbf{e}$ has the following matrix representation

$$
\mathbf{e} \rightarrow P_{1}=\left[\begin{array}{llll}
0 & 1 & 0 & 0 \\
0 & 0 & 1 & 0 \\
0 & 0 & 0 & 1 \\
1 & 0 & 0 & 0
\end{array}\right]
$$

Let us state

$$
\begin{aligned}
\tau_{0}^{l} & =e_{l}, I=0,1,2,3, \\
\tau_{1}^{\prime} & =e_{l} i \sin s, I=0,1,2,3, \\
\tau_{2}^{\prime} & =e_{l} \cos s, I=0,1,2,3, \\
\tau_{2 k}^{\prime} & =e_{l} \cos k s, \quad \tau_{2 k+1}^{\prime}=e_{l} i \sin (k+1) s, I=0,1,2,3, \\
k & =0,1,2, \ldots
\end{aligned}
$$

Thus,

$$
\begin{aligned}
\tau_{2 n}^{0} \tau_{2 k}^{0} & =\frac{1}{2}\left(\tau_{2(n-k)}^{0}+\tau_{2(n+k)}^{0}\right), n \geq k, \\
\tau_{2 n+1}^{I_{1}} \tau_{2 k+1}^{I_{2}} & =\frac{1}{2}\left(\tau_{2(n-k)}^{I_{1} \oplus I_{2}}-\tau_{2(n+k)}^{I_{1} \oplus I_{2}}\right), n \geq k, \\
\tau_{2 n+1}^{I_{1}} \tau_{2 k}^{I_{2}} & =\frac{1}{2}\left(\tau_{2(n-k)+1}^{I_{1} \oplus I_{2}}+\tau_{2(n+k)+1}^{I_{1} \oplus I_{2}}\right), n \geq k .
\end{aligned}
$$

Let us introduce the following algebra

$$
\mathbf{A}=\left\{\sum_{k=0}^{+\infty} \sum_{l=0}^{3}\left(a_{2 k}^{\prime} \tau_{2 k}^{\prime}+a_{2 k+1}^{\prime} \tau_{2 k+1}^{\prime}\right) \mid a_{j}^{\prime} \in \mathbb{R}\right\}
$$

where $\sum_{k=0}^{+\infty} \sum_{l=0}^{3}\left(\left|a_{2 k}^{l}\right|^{2}+\left|a_{2 k+1}^{l}\right|^{2}\right)<+\infty$. It is easily verified that $\mathbf{A}$ is commutative. 
We consider the subspace $\mathbf{B}=\left\{a_{0} \tau_{1}^{1}+a_{1} \tau_{2}^{1}+a_{2} \tau_{0}^{0} \mid a_{i} \in \mathbb{R}\right\}$ of the algebra $\mathbf{A}$.

Let us introduce the function

$$
\mathbf{f}: \mathbf{B} \rightarrow \mathbf{A}
$$

$\left(\mathbf{f}(t, y, z)=f\left(e_{1}(t \cos s+y i \sin s)+z\right)\right)$ as follows

$$
\mathbf{f}(t, y, z)=\sum_{k=0}^{+\infty} \sum_{l=0}^{3}\left(v_{2 k}^{l}(t, y, z) \tau_{2 k}^{l}+v_{2 k+1}^{l}(t, y, z) \tau_{2 k+1}^{l}\right)
$$

The function $\mathbf{f}$ is called $\mathbf{B} / \mathbf{A}$ differentiable at $\mathbf{x}_{0} \in \mathbf{B}$ if there exists $\mathbf{f}^{\prime}\left(\mathbf{x}_{0}\right) \in \mathbf{A}$ such that for any $\mathbf{h} \in \mathbf{B}$,

$$
\mathbf{f}^{\prime}\left(\mathbf{x}_{0}\right) \mathbf{h}=\lim _{\varepsilon \rightarrow 0} \frac{\mathbf{f}\left(\mathbf{x}_{0}+\varepsilon \mathbf{h}\right)-\mathbf{f}\left(\mathbf{x}_{0}\right)}{\varepsilon}
$$

It is easily seen that if $\mathbf{f}$ is $\mathbf{B} / \mathbf{A}$ differentiable, then

$$
\frac{\partial}{\partial t} \mathbf{f}=e_{1} \cos s \frac{\partial}{\partial z} \mathbf{f}
$$

and

$$
\frac{\partial}{\partial y} \mathbf{f}=e_{1} i \sin s \frac{\partial}{\partial z} \mathbf{f}
$$

In this case, all $v_{2 k}^{l}(t, y, z)$ are solutions of Equation (29). Indeed,

$$
\left(\frac{\partial^{2}}{\partial t^{2}}-\frac{\partial^{2}}{\partial y^{2}}\right)^{2} \mathbf{f}-\frac{\partial^{4}}{\partial z^{4}} \mathbf{f}=e_{1}^{4}\left(\cos ^{2} s-(i \sin s)^{2}\right)^{2}-1=0
$$

In the sequel, we denote by e the element $e_{1}$.

We will seek for a solution of Equation (29) in the following form

$$
g_{c}(\mathbf{e}(t \cos s+y i \sin s))=e^{\mathbf{e}(t \cos s+y i \sin s)} .
$$

Because $f(\mathbf{e}(t \cos s+y i \sin s)+z)=g_{c}(\mathbf{e}(t \cos s+y i \sin s)) e^{z}$, we have

$$
v_{k}^{\prime}(t, y, z)=u_{k}^{\prime}(t, y) e^{z}, I=0,1,2,3, k=0,1,2, \ldots,
$$

where $g_{c}(t, y)=\sum_{k=0}^{+\infty} \sum_{l=0}^{3}\left(u_{2 k}^{l}(t, y) \tau_{2 k}^{l}+u_{2 k+1}^{l}(t, y) \tau_{2 k+1}^{\prime}\right)$.

Therefore, we obtain functions $u_{0}^{l}(t, y)$ for $t \geq|y|$ from the following equation

$$
\sum_{l=0}^{3} u_{0}^{l}(t, y) \tau_{0}^{l}=\sum_{l=0}^{3} u_{0}^{l}(t, y) \mathbf{e}^{l}=\frac{1}{2 \pi} \int_{-\pi}^{\pi} e^{\mathbf{e}(t \cos s+y i \sin s)} d s=J_{0}\left(\mathbf{e} i \sqrt{y^{2}-t^{2}}\right)=I_{0}\left(\mathbf{e} \sqrt{t^{2}-y^{2}}\right),
$$

where $I_{k}$ (respectively, $J_{k}$ ) is the modified Bessel (respectively, Bessel) function of the first kind and $k$ th order.

It follows from Equations (30) and (31), the following Cauchy-Riemann type conditions

$$
\begin{aligned}
\frac{\partial}{\partial t} u_{0}^{\prime \oplus 1} & =\frac{1}{2} u_{2}^{\prime}, \\
\frac{\partial}{\partial t} u_{1}^{\prime \oplus 1} & =\frac{1}{2} u_{3}^{\prime}, \\
\frac{\partial}{\partial t} u_{2}^{\prime \oplus 1} & =u_{0}^{l}+\frac{1}{2} u_{4,}^{\prime} \\
\frac{\partial}{\partial t} u_{2 k-1}^{\prime \oplus 1} & =\frac{1}{2}\left(u_{2 k-3}^{l}+u_{2 k+1}^{l}\right), \\
\frac{\partial}{\partial t} u_{2 k}^{\prime \oplus 1} & =\frac{1}{2}\left(u_{2(k-1)}^{l}+u_{2(k+1)}^{l}\right), \\
k & =2,3, \ldots ;
\end{aligned}
$$


and

$$
\begin{aligned}
\frac{\partial}{\partial y} u_{0}^{\prime \oplus 1} & =-\frac{1}{2} u_{1}^{\prime}, \\
\frac{\partial}{\partial y} u_{1}^{\prime \oplus 1} & =u_{0}^{l}-\frac{1}{2} u_{4,}^{\prime} \\
\frac{\partial}{\partial y} u_{2}^{\prime \oplus 1} & =-\frac{1}{2} u_{3}^{\prime}, \\
\frac{\partial}{\partial y} u_{2 k+1}^{\prime \oplus 1} & =\frac{1}{2}\left(u_{2 k}^{l}-u_{2(k+2)}^{\prime}\right), \\
\frac{\partial}{\partial y} u_{2 k+2}^{\prime \oplus 1} & =\frac{1}{2}\left(u_{2 k-1}^{\prime}-u_{2 k+3}^{l}\right), \\
k & =1,2, \ldots
\end{aligned}
$$

By using Equations (32) and (33) and functions $u_{0}^{l}(t, y)$, we can obtain recursively function $u_{k}^{l}(t, y)$ for any $k \geq 1$, which will be used for solution of Equation (28).

Taking into account that $g_{c}(\mathbf{e}(t \cos s+y i \sin s))=e^{\mathbf{e}(t \cos s+y i \sin s)}$, we have

$$
u_{0}^{0}(t, y)+\mathbf{e} u_{0}^{1}(t, y)+\mathbf{e}^{2} u_{0}^{2}(t, y)+\mathbf{e}^{3} u_{0}^{3}(t, y) \quad=\frac{1}{2 \pi} \int_{-\pi}^{\pi} e^{\mathbf{e}(t \cos s+y i \sin s)} d s=I_{0}\left(\mathbf{e} \sqrt{t^{2}-y^{2}}\right) .
$$

It is easily seen that

$$
\begin{aligned}
I_{0}\left(\mathbf{e} \sqrt{t^{2}-y^{2}}\right) & =\frac{I_{0}\left(\sqrt{t^{2}-y^{2}}\right)+I_{0}\left(i \sqrt{t^{2}-y^{2}}\right)}{2}+\mathbf{e}^{2}\left(\frac{I_{0}\left(\sqrt{t^{2}-y^{2}}\right)-I_{0}\left(i \sqrt{t^{2}-y^{2}}\right)}{2}\right) \\
& =\frac{I_{0}\left(\sqrt{t^{2}-y^{2}}\right)+J_{0}\left(\sqrt{t^{2}-y^{2}}\right)}{2}+\mathbf{e}^{2} \frac{I_{0}\left(\sqrt{t^{2}-y^{2}}\right)-J_{0}\left(\sqrt{t^{2}-y^{2}}\right)}{2} .
\end{aligned}
$$

Therefore, for $t \geq|y|$, we have $u_{0}^{1}(t, y)=u_{0}^{3}(t, y)=0$ and

$$
\begin{aligned}
& u_{0}^{0}(t, y)=\frac{I_{0}\left(\sqrt{t^{2}-y^{2}}\right)+J_{0}\left(\sqrt{t^{2}-y^{2}}\right)}{2}, \\
& u_{0}^{2}(t, y)=\frac{I_{0}\left(\sqrt{t^{2}-y^{2}}\right)-J_{0}\left(\sqrt{t^{2}-y^{2}}\right)}{2} .
\end{aligned}
$$

It follows from the first two equations of Equation (33) that

$$
\begin{aligned}
& u_{1}^{1}=-2 \frac{\partial}{\partial y} u_{0}^{2}=-\frac{\partial\left[I_{0}\left(\sqrt{t^{2}-y^{2}}\right)-J_{0}\left(\sqrt{t^{2}-y^{2}}\right)\right]}{\partial y}=\frac{y}{\sqrt{t^{2}-y^{2}}}\left(I_{1}\left(\sqrt{t^{2}-y^{2}}\right)+J_{1}\left(\sqrt{t^{2}-y^{2}}\right)\right), \\
& u_{1}^{3}=-2 \frac{\partial}{\partial y} u_{0}^{0}=-\frac{\partial\left[I_{0}\left(\sqrt{t^{2}-y^{2}}\right)+J_{0}\left(\sqrt{t^{2}-y^{2}}\right)\right]}{\partial y}=\frac{y}{\sqrt{t^{2}-y^{2}}}\left(I_{1}\left(\sqrt{t^{2}-y^{2}}\right)-J_{1}\left(\sqrt{t^{2}-y^{2}}\right)\right), \\
& u_{1}^{0}=-2 \frac{\partial}{\partial y} u_{0}^{1}=0, \\
& u_{1}^{2}=-2 \frac{\partial}{\partial y} u_{0}^{3}=0 .
\end{aligned}
$$

Then it follows from the Cauchy-Riemann type conditions (32) that

$$
\begin{aligned}
& u_{2}^{0}(t, y)=2 \frac{\partial u_{0}^{1}(t, y)}{\partial t}=0 \\
& u_{2}^{1}(t, y)=2 \frac{\partial u_{0}^{2}(t, y)}{\partial t}=\frac{\partial\left(I_{0}\left(\sqrt{t^{2}-y^{2}}\right)-J_{0}\left(\sqrt{t^{2}-y^{2}}\right)\right)}{\partial t}=\frac{t}{\sqrt{t^{2}-y^{2}}}\left(I_{1}\left(\sqrt{t^{2}-y^{2}}\right)+J_{1}\left(\sqrt{t^{2}-y^{2}}\right)\right) \\
& u_{2}^{2}(t, y)=2 \frac{\partial u_{0}^{3}(t, y)}{\partial y}=0 \\
& u_{2}^{3}(t, y)=2 \frac{\partial u_{0}^{0}(t, y)}{\partial t}=\frac{\partial\left[I_{0}\left(\sqrt{t^{2}-y^{2}}\right)+J_{0}\left(\sqrt{t^{2}-y^{2}}\right)\right]}{\partial t}=\frac{t}{\sqrt{t^{2}-y^{2}}}\left(I_{1}\left(\sqrt{t^{2}-y^{2}}\right)-J_{1}\left(\sqrt{t^{2}-y^{2}}\right)\right) .
\end{aligned}
$$


Similarly, for $u_{3}^{l}$, we have

$$
\begin{aligned}
u_{3}^{0}= & 2 \frac{\partial}{\partial t} u_{1}^{1}=2 \frac{\partial}{\partial t}\left[\frac{y}{\sqrt{t^{2}-y^{2}}}\left(I_{1}\left(\sqrt{t^{2}-y^{2}}\right)+J_{1}\left(\sqrt{t^{2}-y^{2}}\right)\right)\right]=-\frac{2 t y}{\sqrt{\left(t^{2}-y^{2}\right)^{3}}}\left(I_{1}\left(\sqrt{t^{2}-y^{2}}\right)+J_{1}\left(\sqrt{t^{2}-y^{2}}\right)\right) \\
& +\frac{t y}{t^{2}-y^{2}}\left(I_{0}\left(\sqrt{t^{2}-y^{2}}\right)+I_{2}\left(\sqrt{t^{2}-y^{2}}\right)+J_{0}\left(\sqrt{t^{2}-y^{2}}\right)-J_{2}\left(\sqrt{t^{2}-y^{2}}\right)\right) ; \\
u_{3}^{2}= & 2 \frac{\partial}{\partial t} u_{1}^{3}=2 \frac{\partial}{\partial t}\left[\frac{y}{\sqrt{t^{2}-y^{2}}}\left(I_{1}\left(\sqrt{t^{2}-y^{2}}\right)-J_{1}\left(\sqrt{t^{2}-y^{2}}\right)\right)\right]=-\frac{2 t y}{\sqrt{\left(t^{2}-y^{2}\right)^{3}}}\left(I_{1}\left(\sqrt{t^{2}-y^{2}}\right)-J_{1}\left(\sqrt{t^{2}-y^{2}}\right)\right) \\
& +\frac{2 t y}{t^{2}-y^{2}}\left(I_{0}\left(\sqrt{t^{2}-y^{2}}\right)+I_{2}\left(\sqrt{t^{2}-y^{2}}\right)-J_{0}\left(\sqrt{t^{2}-y^{2}}\right)+J_{2}\left(\sqrt{t^{2}-y^{2}}\right)\right) .
\end{aligned}
$$

It is easily seen that $u_{3}^{1}=u_{3}^{3}=0$.

Next, it follows from Equation (32) that

$$
\begin{aligned}
u_{4}^{0}= & 2 \frac{\partial u_{2}^{1}}{\partial t}-2 u_{0}^{0}=2 \frac{\partial}{\partial t} \frac{t}{\sqrt{t^{2}-y^{2}}}\left(I_{1}\left(\sqrt{t^{2}-y^{2}}\right)+J_{1}\left(\sqrt{t^{2}-y^{2}}\right)\right)-2 u_{0}^{0}=\frac{-2 y^{2}}{\sqrt{\left(t^{2}-y^{2}\right)^{3}}}\left(I_{1}\left(\sqrt{t^{2}-y^{2}}\right)+J_{1}\left(\sqrt{t^{2}-y^{2}}\right)\right) \\
& +\frac{t^{2}}{t^{2}-y^{2}}\left(I_{0}\left(\sqrt{t^{2}-y^{2}}\right)+I_{2}\left(\sqrt{t^{2}-y^{2}}\right)+J_{0}\left(\sqrt{t^{2}-y^{2}}\right)-J_{2}\left(\sqrt{t^{2}-y^{2}}\right)\right)-I_{0}\left(\sqrt{t^{2}-y^{2}}\right)-J_{0}\left(\sqrt{t^{2}-y^{2}}\right) ; \\
u_{4}^{2}= & 2\left(\frac{\partial u_{2}^{3}}{\partial t}-u_{0}^{2}\right)=\frac{-2 y^{2}}{\sqrt{\left(t^{2}-y^{2}\right)^{3}}}\left(I_{1}\left(\sqrt{t^{2}-y^{2}}\right)-J_{1}\left(\sqrt{t^{2}-y^{2}}\right)\right) \\
& +\frac{t^{2}}{t^{2}-y^{2}}\left(I_{0}\left(\sqrt{t^{2}-y^{2}}\right)+I_{2}\left(\sqrt{t^{2}-y^{2}}\right)-J_{0}\left(\sqrt{t^{2}-y^{2}}\right)+J_{2}\left(\sqrt{t^{2}-y^{2}}\right)\right)-I_{0}\left(\sqrt{t^{2}-y^{2}}\right)+J_{0}\left(\sqrt{t^{2}-y^{2}}\right) .
\end{aligned}
$$

In addition, it is easily verified that $u_{4}^{1}=u_{4}^{3}=0$.

Continuing this process, we can calculate $u_{k}^{m}, m=0,1,2,3$ for any $k \geq 1$. The number of $u_{k}^{m}$ in the extension of a solution of the equation depends on the initial conditions for the solution.

\section{Acknowledgements}

This work was partially supported by the State fund for fundamental researches of Ukraine, project no. 25.1/084. We also thank Tecnologico de Monterrey for the support provided in the development of this work through the Research Chair in Telecommunications. The third-named author was partially supported by CONACyT projects as well as by Instituto Politécnico Nacional in the framework of SIP and COFAA programs.

\section{References}

1. Ketchum PW. A complete solution of Laplace's equation by an infinite hypervariable. American Journal of Mathematics 1929; 51:179-188.

2. Ketchum PW. Analytic functions of hypercomplex variables. Transactions of the American Mathematical Society 1928; 30:641-667.

3. Mel'nichenko IP. Biharmonic bases in algebras of the second rank. Ukrainian Mathematical Journal 1986; 40(8):224-226.

4. Kovalev VF, Mel'nichenko IP. Biharmonic functions on a biharmonic plane. Doklady Academii Nauk UkrSSR (Reports of the Academy of Sciences of the Ukrainian SSR) 1988; 8:25-27. (In Russian).

5. Kunz KZ. Application of an algebraic technique to the solution of Laplace's equation in three dimensions. SIAM Journal on Applied Mathematics 1971; 21(3):425-441.

6. Rochon D, Shapiro M. On algebraic properties of bicomplex and hyperbolic numbers. Analele Universitatii din Oradea. Fascicola Matematica 2004; 11:1-28.

7. Casas F. Solution of linear partial differential equations by Lie algebraic methods. Journal of Computational and Applied Mathematics 1996; 76:159-170.

8. Krasnov Y. Differential equations in algebras. In Hypercomplex Analysis, Sabadini I, Shapiro M, Sommen F (eds), Trends in Mathematics. Birkhäuser: Basel, 2009; 187-205.

9. Pogorui AA, Rodríguez-Dagnino R. M. One-dimensional semi-Markov evolutions with general Erlang sojourn times. Random Operators and Stochastic Equations 2005; 13(4):399-405.

10. Pogorui AA. Monogenic functions on commutative algebras. Complex Variables and Elliptic Equations 2007; 52(12):1155-1159.

11. Kravchenko VV, Rochon D, Tromblay S. On the Klein-Gordon equation and hyperbolic pseudoanalytic function theory. Journal of Physics A: Mathematical and Theoretical 2008; 065205.

12. Fushchych WI, Roman OV, Zhdanov RZ. Symmetry reduction and exact solutions of nonlinear biwave equations. Reports on Mathematical Physics 1996; 37(2):267-281.

13. Yaglom IM. Complex Numbers in Geometry. Academic Press Inc.: U.S.A, 1968. 